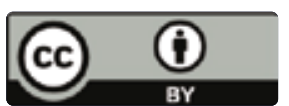

\title{
CARACTERÍSTICAS EPIDEMIOLÓGICAS, CLÍNICAS Y TERAPÉUTICAS DE LA INFECCIÓN URINARIA EN PACIENTES DIABÉTICOS
}

\author{
EPIDEMIOLOGICAL, CLINICAL AND THERAPEUTIC CHARACTERISTICS OF \\ URINARY INFECTION IN DIABETIC PATIENTS
}

\author{
Indira Arizu Cortegana-Venegas ${ }^{1, a}$. \\ 1. Médico cirujano. \\ a. Segunda Especialidad de la Facultad de Medicina. Universidad Nacional San Luis Gonzaga de Ica, Perú
}

Correspondencia:

Indira Arizu Cortegana Venegas Dirección: Jiron Hermanos Angulo No 333 - Santa Rosa Pueblo Nuevo Chincha.

Número de celular: 943825709. Correo Electrónico:

indiravenegas@hotmail.com/ indiravenegas@yahoo.com

\section{Contribuciones De Autoría:}

IACV: Contribuciones en la concepción y diseño del manuscrito, recopilación, análisis e interpretación de la información, expresión y revisión exhaustiva del contenido del manuscrito y aprobación final del artículo.

Conflicto De Intereses: Los autores declaran no tener conflictos de interé Financiamiento: Autofinanciado.

\section{Como Citar}

Cortegana-Venegas I. Características epidemiológicas, clínicas y terapéuticas de la infección urinaria en pacientes diabéticos. Rev méd panacea. 2020; 9(1): 43-49. doi: https://doi.org/10.35563/rmp.v9i1.296

Recibido: $11-01-2020$

Aceptado: 16 - $02-2020$

Publicado: 06 - 03 - 2020

\section{RESUMEN}

Introducción: La infección urinaria en diabéticos constituye uno de los problemas importantes caracterizado por su unidad clínica y pluralidad etiológica. Objetivo: Generar conocimiento sobre las características, epidemiológicas, clínicas y terapéuticas de infección urinaria en diabéticos. Materiales y métodos: Es un estudio descriptivo de búsqueda bibliografía y se ha realizado en Pubmed, Medline, Scielo, bibliotecas de universidades nacionales e internacionales. Resultados: El $40.74 \%$ de casos fueron varones y $59.26 \%$ mujeres. Los malos hábitos de higiene, la presencia de cálculos renales, un tiempo de enfermedad mayor de 10 años, vejiga neurogénica, uso de corticoides, infección urinaria previa están asociadas a infección urinaria en la población de diabéticos. Casi el 30\% de los pacientes presentaron bacteriuria asintomática. El síntoma más frecuente fue la fiebre. Los síntomas presentes en el 75,7\%. La incontinencia de esfuerzo $45.3 \%$, de urgencia $40.6 \%$, síntomas obstructivos $25 \%$, irritativos $10.1 \%$. Predominó Escherichia coli $(57.41 \%)$, seguido de Enterobacter (8.33\%) y Klebsiella pneumoniae (6.48\%). La resistencia de E. coli fue elevada contra clindamicina, ácido nalidíxico, ácido pipemídico (100\%) y cefuroxima $(90.91 \%)$, amoxicilina clavulanato (81.25\%), ampicilina (78.57\%) y cefalotina (72.22\%); buena sensibilidad para imipenem $(76.92 \%)$, cefepime $(72.73 \%)$, amikacina $(72.41 \%)$, nitrofurantoína $(70.37 \%)$, ceftriaxona (63.79\%) y ceftazidima (61.11\%). Conclusión: Las infecciones urinarias en los pacientes diabéticos son frecuentes y ocasionadas con más frecuencia por $\mathrm{E}$. coli, con patrones de resistencia y sensibilidad que requieren medidas de intervención. La resistencia a los antibióticos se incrementa por el uso indiscriminado en pacientes con inadecuado control de su enfermedad.

Palabras clave: Características, infección urinaria, diabetes, epidemiología.

\section{ABSTRACT}

Introduction: Urinary infection in diabetics is one of the important problems characterized by its clinical unit and etiological plurality. Objective: To generate knowledge about the characteristics, epidemiological, clinical and therapeutic of urinary infection in diabetics. Materials and methods: It is a descriptive study of literature search and has been carried out in Pubmed, Medline, Scielo, libraries of national and international universities. Results: $40.74 \%$ of cases were male and $59.26 \%$ female. Bad hygiene habits, the presence of kidney stones, a disease time of more than 10 years, neurogenic bladder, corticosteroid use, previous urinary infection is associated with urinary infection in the diabetic population. Almost $30 \%$ of the patients presented asymptomatic bacteriuria. The most frequent symptom was fever. The symptoms present in $75.7 \%$. Stress incontinence $45.3 \%$, emergency $40.6 \%$, obstructive symptoms $25 \%$, irritative $10.1 \%$. Escherichia coli (57.41\%) predominated, followed by Enterobacter (8.33\%) and Klebsiella pneumoniae (6.48\%). The resistance of E. coli was high against clindamycin, nalidixic acid, pipemidic acid (100\%) and cefuroxime (90.91\%), amoxicillin clavulanate (81.25\%), ampicillin (78.57\%) and cephalothin (72.22\%); Good sensitivity for imipenem (76.92\%), cefepime (72.73\%), amikacin $(72.41 \%)$, nitrofurantoin (70.37\%), ceftriaxone (63.79\%) and ceftazidime (61.11\%). Conclusions: Urinary infections in diabetic patients are frequent and more frequently caused by $\mathrm{E}$. coli, with resistance and sensitivity patterns that require intervention measures. Antibiotic resistance is increased by indiscriminate use in patients with inadequate control of their disease.

Keywords: Characteristics, urinary tract infection, diabetes, epidemiology. 


\section{INTRODUCCIÓN}

En pacientes diabéticos, las infecciones del tracto urinario pueden representar una amenaza grave en su estado de salud, ya que éstas pueden favorecer la aparición de bacteriemia, cistitis o pielonefritis enfisematosa. En pacientes con diagnóstico crónico de diabetes mellitus sin control adecuado, es común observar una pérdida de sensibilidad en diversas áreas corporales debido al daño en el tejido nervioso, originando entonces infección del tracto urinario asintomáticas (1).

Se evidencia en diferentes investigaciones que existe mayor prevalencia de infección urinaria en diabéticos respecto a los no diabéticos; en estudio realizado en el Reino Unido mostró que la tasa de incidencia de infección urinaria fue de 46,9 por 1.000 personas al año en pacientes diabéticos y 29,9 para los pacientes sin diabetes. Por sus condiciones anatómicas las mujeres presentan mayor prevalencia de infección urinaria respecto a los hombres; $12,9 \%$ (4.517pacientes) y 3,9\% (1.497 pacientes) respectivamente en un estudio en Estados Unidos. Otro estudio que se llevó a cabo entre 2011 y 2014 en Kuwait e incluyeron a 252 diabéticos con infección urinaria, establecieron que los diabéticos tienen mayor riesgo de infección urinaria en particular en las mujeres $(88,5 \%)(2)$.

El microorganismo más común en diabéticos con infección urinaria es E. coli (64\%), una frecuencia menor a la de la población general, en tanto que las causadas por Klebsiella pneumoniae son más comunes en los diabéticos que en la población general. Otros microorganismos son Proteus spp., Enterobacter spp. y estafilococos negativos para coagulasa. Los pacientes diabéticos presentan con mayor frecuencia infecciones de las vías urinarias causadas por bacterias inusuales, como Acinetobacter spp. y estreptococos del grupo B; además, dicha enfermedad es uno de los factores de riesgo principales para infecciones urinarias de origen fúngico, especialmente las causadas por Candida spp. (3).

La mala circulación en los diabéticos, la reducción de la capacidad de los glóbulos blancos para combatir infecciones, las vejigas disfuncionales que se contraen mal pueden contribuir al aumento prevalencia de infección urinaria en individuos diabéticos. Las complicaciones diabéticas, como la neuropatía y la glucosuria, también pueden predisponer a tales infecciones (4).

La infección urinaria abarca una variedad de entidades clínicas, que incluyen bacteriuria asintomática, cistitis, prostatitis y pielonefritis. Puede haber un mayor riesgo de bacteriuria asintomática o infección urinaria sintomática en diabéticos. La infección urinaria no complicada puede ser bacteriuria asintomática, cistitis, pielonefritis, en hombres y mujeres no embarazadas, también prostatitis en hombres. La infección urinaria complicada puede ser episodios sintomáticos de cistitis o pielonefritis en hombres o mujeres con una predisposición anatómica a la infección, con un cuerpo extraño en el tracto urinario, o factores que predisponen a una respuesta tardía al tratamiento. Puede haber una recaída o reinfección (5).

El creciente problema de resistencia a antimicrobianos ha hecho que las infecciones urinarias necesiten ahora tratamiento parenteral, lo que impone costos adicionales al sistema de salud y malestar al paciente; es una amenaza significativa de salud pública. El desarrollo de resistencia es acelerado por la presión selectiva del uso de agentes antimicrobianos. La automedicación de los pacientes, facilitada por la venta libre de fármacos antimicrobianos, empeora el problema. La terapia antibiótica para tratar las infecciones urinarias en pacientes con diabetes debería basarse, siempre que sea posible, en el urocultivo y el antibiograma (6).

El objetivo principal de esta revisión bibliográfica es generar conocimiento sobre las características, epidemiológicas, clínicas y terapéuticas de infección urinaria pacientes diabéticos.

\section{MATERIALES Y MÉTODOS}

La búsqueda bibliográfica se ha realizado en Pubmed, Medline, Scielo, bibliotecas de universidades nacionales e internacionales. Como criterios de selección se tomó en cuenta información publicada en dichas fuentes dada la confiabilidad de las mismas, con una antigüedad no mayor de 5 años.

\section{RESULTADOS.}

Lucas E. Y Col. (Ecuador 2019). Infección urinaria en pacientes con diabetes mellitus tipo 2: Frecuencia, etiología, susceptibilidad antimicrobiana. Se estudiaron 108 pacientes ambulatorios, con diagnóstico presuntivo de infección de vías urinarias, durante el periodo mayo 2016-mayo 2017, en la ciudad de Jipijapa, Ecuador. Para el urocultivo se utilizó la técnica del asa calibrada. La susceptibilidad a los antibióticos se determinó mediante el método de Kirby \& Bauer. Las variables cualitativas se compararon con ji cuadrado o la prueba exacta de Fisher. Se calculó el Odd radio (OR) y sus intervalos de confianza (IC) del 95\%. La frecuencia de infección urinaria fue de $73,15 \%$. El microorganismo más frecuentemente aislado fue Escherichia coli (78,48\%). Los mayores porcentajes de resistencia se observaron para amoxicilina (78,87\%) y cefalexina $(71,83 \%)$. Los malos hábitos de higiene, la presencia de cálculos renales y una vida sexual activa resultaron factores relacionados para las infecciones urinarias. La nitrofurantoina, fosfomicina, fluoroquinolonas y algunos betalactámicos, todavía representan una alternativa de utilidad en la quimioterapia de las infecciones urinarias no complicadas en pacientes diabéticos (7).

Nocua LC. Y Col. (Colombia 2017). Perfil de sensibilidad antimicrobiana de microorganismos causantes de 
infecciones urinarias en pacientes con diabetes mellitus en Colombia. Se hizo un estudio descriptivo de un subgrupo de pacientes diabéticos en el marco de una investigación en adultos con infección de las vías urinarias. Durante un año, se recolectaron aislamientos de Escherichia coli, Klebsiella spp. y Proteus mirabilis en nueve hospitales de Colombia y se determinó su perfil de sensibilidad. Se recolectaron 68 aislamientos (58 de E. coli, nueve de Klebsiella spp. y uno de P. mirabilis). Cuatro (6,9 $\%)$ de los aislamientos de E. coli expresaron dichas betalactamasas, en dos (3,4\%) de ellos, pertenecientes al grupo filogenético B2 y al clon ST131, se detectaron las betalactamasas TEM-1 y CTM-X-15. En otros cuatro $(6,9$ $\%)$ aislamientos de $E$. coli se encontró el fenotipo AmpC, y en tres de ellos se produjeron las betalactamasas TEM-1 y CMY-2. Un aislamiento de K. pneumoniae expresó la carbapenemasa KPC-3. Se confirmó la presencia de cepas productoras de betalactamasas de espectro extendido y carbapenemasas en microorganismos responsables de infección urinaria en pacientes diabéticos (8).

Erazo PN. Y Col. (Ecuador 2018). Características de resistencia bacteriana a los antibióticos en pacientes con Diabetes Mellitus tipo 2 con infección del tracto urinario en el servicio de clínica del Hospital "Luis F. Martínez" durante el período 2012-2017. Estudio cuantitativo descriptivo, universo conformado por 400 historias clínicas, la muestra fue de 196. La frecuencia de infección del tacto urinario en pacientes con diabetes fue del $100 \%$. El $58,5 \%$ de urocultivos obtuvo aislamiento microbiológico y Escherichia Coli prevaleció (41,8\%). Existe resistencia al trimetropin sulfametoxazol (66,1\%), la nitrofurantoína (70\%) y quinolonas (70,5\%) aún mantienen sensibilidad. El uso de aminopenicilinas se incrementó $(14,2 \%)$ como tratamiento posterior. Un $20,4 \%$ de los pacientes al ingreso se mantuvieron sin tratamiento. El 57,6\% mantuvieron el mismo esquema tras resultados de laboratorio y el $42,3 \%$ sufrió cambios. Los pacientes de 40 años y más, de estado civil unión de hecho y viudez, sin instrucción y con mal control metabólico presentaron alto niveles de resistencia, el sexo masculino $(54,5 \%)$ y femenino $(72,7 \%)$ presentaron resistencia a macrólidos y trimetropin sulfametoxazol respectivamente. La resistencia a los antibióticos se incrementa por el uso indiscriminado en pacientes mayores y con inadecuado control de su enfermedad (9).

Aswami SM. Y Col. (Australia 2014). Clinical profile of urinary tract infections in diabetics and non-diabetics. Se estudiaron un total de 181 diabéticos (83 hombres y 98 mujeres) y 124 sujetos no diabéticos (52 hombres y 72 mujeres) con UTI de cultivo positivo. Se excluyeron los pacientes con cultivo de orina negativo $(n=64)$, aquellos diagnosticados y tratados fuera $(n=83)$ y que no estaban dispuestos a participar en el estudio $(n=24)$. Casi el 30 por ciento de los pacientes (diabéticos y no diabéticos) se presentaron con bacteriuria asintomática y la prevalencia de pielonefritis fue significativamente mayor $(p=0,04)$ en los diabéticos en comparación con los pacientes no diabéticos. La mayoría de los diabéticos con IU (87,14 por ciento) tenían hemoglobina glicosilada $(\mathrm{HbA} 1 \mathrm{c})>6,5$ por ciento con $p<0,001$. La tasa de aislamiento de Escherichia coli (E. coli) en el cultivo de orina fue mayor $(64,6 \%)$ entre los pacientes diabéticos, seguida de Klebsiella $(12,1 \%)$ y Enterococcus (9,9\%). La prevalencia de espectro extendido de betalactamasa (BLEE) que produce $E$. coli fue significativamente mayor en los diabéticos ( $p=0,001)$ en comparación con los no diabéticos. E.coli mostró una sensibilidad máxima a los carbapenems en sujetos diabéticos y no diabéticos y una menor susceptibilidad a la ampicilina. La prevalencia de pielonefritis es significativamente mayor en los diabéticos que en los sujetos no diabéticos, siendo E. coli el aislado más común. La hemoglobina glicosilada elevada ( $\mathrm{HbA} 1 \mathrm{c})$ predispone a los diabéticos a la ITU. La investigación de la bacteriuria en pacientes diabéticos para la infección del tracto urinario es importante para el tratamiento y la prevención de complicaciones renales (10).

Garg V. Y Col. (India 2015). Comparison of Clinical Presentation in Diabetic and Non-Diabetic Females with Urinary Tract Infection Assessed as Per the European Association of Urology Classification. Este es un estudio prospectivo de un solo centro realizado durante un período de un año en Dayanand Medical College and Hospital en un total de 151 pacientes diabéticas (Grupo A) y no diabéticas (Grupo B) con diagnóstico de IU. La ITU se clasificó de acuerdo con la clasificación de la EAU para la ITU, y se hizo un esfuerzo para averiguar la clase frecuente de ITU en este grupo de estudio. Se estudiaron un total de 151 mujeres que incluyeron 70 mujeres diabéticas (Grupo A) y 81 mujeres no diabéticas (Grupo B). El síntoma más frecuente fue fiebre en ambos grupos. La ITU se clasificó según los grados de EAU de la ITU. La presentación clínica más común en ambos grupos fue la cistitis seguida de pielonefritis y urosepsis. El organismo más común fue la E-coli, que era susceptible a la mayoría de los antibióticos. Las infecciones urinarias en pacientes diabéticas y no diabéticas tienen patrones diferentes. La diabetes no controlada se asoció más comúnmente con una IU grave, como la pielonefritis y la pielonefritis enfisematosa. E. coli fue el aislado más común en cualquiera de los grupos, seguido por klebsiella y Pseudomonas. Candida fue aislada solamente de la población diabética (11).

Tovar H. Y Col (Colombia 2016). Infección del tracto urinario en pacientes hospitalizados con diabetes tipo 2. Revisión de una base de datos de pacientes diabéticos mayores de 18 años, ingresados en un Servicio de Medicina en Colombia. Se revisaron los datos de 470 pacientes de $65 \pm 13$ años (50\% mujeres). Se detectaron infecciones del tracto urinario en 68 (14\%), todas adquiridas en la comunidad. Se realizó un cultivo en 50 (73\%), que fue positivo en $80 \%$. Los microorganismos más comunes aislados fueron Escherichia coli y Klebsiella pneumoniae en 52 y $18 \%$ respectivamente. $28 \%$ de las cepas de E. coli fueron resistentes a las betalactamasas de espectro extendido y el $14 \%$ de las cepas de $\mathrm{K}$. pneumoniae fueron resistentes a la ampicilina. Se observaron complicaciones en el $28 \%$ de estos pacientes 
y el $6 \%$ falleció, principalmente por shock séptico. Los niveles de hemoglobina glicosilada en pacientes con y sin infección del tracto urinario fueron 9.2 y $8.5 \%$ respectivamente $(p=0.016)$. La infección del tracto urinario es común entre los pacientes diabéticos hospitalizados y se asocia con complicaciones y mayor mortalidad (12).

Jiménez J. Y Col. (México 2016). Prevalencia de síntomas urinarios en mujeres con diabetes mellitus. Se evaluó una cohorte de mujeres del programa DiabetIMSS de enero de 2011 a 2013. Se evaluaron un total de 169 mujeres. La mediana de edad fue de 58 años (29-85) y el tiempo de evolución principal de la DM fue de 9 años $(0,5-31)$. Los síntomas del tracto urinario estaban presentes en 128 $(75,7 \%)$ pacientes. La incontinencia de esfuerzo y de urgencia estuvo predominantemente presente (45.3 y $40.6 \%$ respectivamente), seguida por síntomas obstructivos e irritativos ( 25 y $10.1 \%$ respectivamente). El impacto en la calidad de vida fue leve-moderado en el $91,1 \%$ de los pacientes. Se observó al menos un criterio para la neuropatía en 154 pacientes (91,1\%). El tiempo de evolución de la neuropatía fue mayor en el grupo sintomático (12 frente a 4,8 meses). Los síntomas estaban presentes principalmente en pacientes con más de un año de neuropatía; $p<0.05$. Existe una alta prevalencia de síntomas del tracto urinario en mujeres diabéticas. El único factor de riesgo asociado fue la neuropatía. No se encontró asociación significativa entre el resto de los factores (13).

Batista A. Y Col. (República Dominicana 2013). Frecuencia de infección de vías urinarias en pacientes diabéticos asistidos en un centro rural. Se realizó un estudio descriptivo, retrospectivo de corte transversal. La población de estudio estuvo constituida por 180 pacientes que se presentaron en este centro de los cuales obtuvimos una muestra de 98 pacientes. De 180 pacientes con diabetes, 98 presentaron infección de vías urinarias. Los pacientes fueron diagnosticados por clínica y pruebas de laboratorio. El sexo más afectado fue el femenino con 54 casos para un 52.92\%. Se observó que los pacientes con más de 5 años de haberse diagnosticado como diabéticos fueron más propensos a sufrir de infección de vías urinarias. Pacientes con presencia de bacteriuria en pruebas analíticas $80 \%$ de los pacientes fueron sintomáticos de infección urinaria (78 casos). Los pacientes diabéticos son más propensos a padecer infección de vías urinarias que un paciente no diabético. Es más común en mujeres que en hombres, esto puede deberse a infecciones vaginales. Se observó que los pacientes con más de 5 años de haberse diagnosticado como diabéticos fueron ligeramente más propensos a sufrir de infección de vías urinarias. No todos los pacientes diabéticos con infección de vías urinarias tienen síntomas (14)

Ríos MV. (Perú 2018). Características sociodemográficas, clínicas y antecedentes patológicos asociados a infección del tracto urinario en diabéticos: estudio de casos y controles. Estudio Comparativo, transversal, retrospectivo y explicativo, realizado en el Hospital Loayza el año 2016. Se revisaron historias de 163 pacientes diabéticos con ITU y 163 diabéticos sin ITU. Las características sociodemográficas no tienen relación estadística con la ocurrencia de ITU ( $p>0.05$ ). Tiempo de Enfermedad $>=10$ años (OR=3.978 (I.C.95\% 1.467-10.790); $p=0.007)$, Insuficiencia renal crónica $(\mathrm{OR}=8.412 \quad$ (I.C. 95\% 1.832-38.615), $p=0.006)$, Litiasis renal $(O R=10.604$ (I.C.95\% 2.144-52.459), $p=0.004)$, Vejiga Neurogénica (OR=12.290 (I.C.95\% 1.447-104.386), $p=0.022)$, Uso de corticoides (OR=17.850 (I.C.95\% 3.789-84.087), $p=0.000)$, ITU previa (OR=27.757 (I.C.95\% 9.280-83.027), $p=0.000)$ y Presentar infección vaginal o patología prostática (OR=4.958 (I.C.95\% 2.149-11.439), $p=0.000)$, estuvieron asociados de forma independiente con la ocurrencia de ITUs. Las características sociodemográficas no tienen relación estadística con ITU. Un tiempo de enfermedad $>=10$ años, la Insuficiencia renal crónica, Litiasis renal, Vejiga Neurogénica, Uso de corticoides, ITU previa y presentar infección vaginal o patología prostática están asociadas estadísticamente con la ocurrencia de ITU en la población de diabéticos (15).

Coila J. (Perú 2015). Etiología y susceptibilidad antimicrobiana de uropatógenos en pacientes diabéticos con infección urinaria en el Hospital May. Od. Julio Pinto Manrique, Arequipa, 2014. Revisión de historias clínicas de pacientes diabéticos que cumplieron criterios de selección; se muestran resultados mediante estadística descriptiva. De 1503 atenciones, en 108 casos se diagnosticó infección urinaria. El $40.74 \%$ de casos fueron varones y $59.26 \%$ mujeres, con edades comprendidas entre los 60 y 79 años (61.11\%). El 25\% de gérmenes fueron gram positivos, principalmente estafilococos (24.07\%) y un caso de estreptococo viridans (0.93\%). El $75 \%$ de gérmenes fueron gram negativos, de los cuales predominó Escherichia coli (57.41\%), seguido de Enterobacter (8.33\%) y Klebsiella pneumoniae (6.48\%); se aislaron dos cepas de Pseudomonas y un caso de Citrobacter $(0.93 \%)$. La resistencia de E. coli fue elevada contra clindamicina, ácido nalidíxico, ácido pipemídico $(100 \%)$ y cefuroxima $(90.91 \%)$, amoxiciina clavulanato (81.25\%), ampicilina (78.57\%) y cefalotina (72.22\%), aunque mantiene buena sensibilidad para imipenem (76.92\%), cefepime (72.73\%) y amikacina (72.41\%) y nitrofurantoína (70.37\%), así como a ceftriaxona (63.79\%) y ceftazidima $(61.11 \%)$ entre otros. Las infecciones urinarias en los pacientes diabéticos son frecuentes y ocasionadas por E. coli, con patrones de resistencia y sensibilidad que requieren medidas de intervención (16).

\section{DISCUSIÓN.}

Los pacientes diabéticos son más propensos a padecer infección de vías urinarias que un paciente no diabético. El $40.74 \%$ de casos fueron varones y $59.26 \%$ mujeres, con edades comprendidas entre los 60 y 79 años un $61.11 \%$ $(14,16)$ 
Los malos hábitos de higiene, la presencia de cálculos renales y una vida sexual activa resultaron factores relacionados para las infecciones urinarias. Un tiempo de enfermedad mayor de 10 años, la Insuficiencia renal crónica, Litiasis renal, Vejiga Neurogénica, Uso de corticoides, infección urinaria previa y presentar infección vaginal o patología prostática están asociadas estadísticamente con la ocurrencia de infección urinaria en la población de diabéticos $(7,14,15)$.

Casi el 30\% de los pacientes (diabéticos y no diabéticos) se presentaron con bacteriuria asintomática y la prevalencia de pielonefritis fue significativamente mayor en los diabéticos en comparación con los pacientes no diabéticos. El síntoma más frecuente fue fiebre en ambos grupos. La presentación clínica más común en ambos grupos. Los síntomas del tracto urinario estaban presentes en el $75,7 \%$ de pacientes diabéticos. La incontinencia de esfuerzo $45.3 \%$ y de urgencia $40.6 \%$ predominantemente presente, seguida por síntomas obstructivos $25 \%$ e irritativos $10.1 \%(10,11,13)$.

Las infecciones urinarias en pacientes diabéticas y no diabéticas tienen patrones diferentes. La diabetes no controlada se asoció más comúnmente con una infección urinaria grave, como la pielonefritis y la pielonefritis enfisematosa (11).

El 75\% de gérmenes fueron gram negativos, de los cuales predominó Escherichia coli (57.41\%), seguido de Enterobacter (8.33\%) y Klebsiella pneumoniae (6.48\%). El $25 \%$ de gérmenes fueron gram positivos, principalmente estafilococos (24.07\%) y un caso de estreptococo viridans $(0.93 \%)(12,16)$.

La resistencia de E. coli fue elevada contra clindamicina, ácido nalidíxico, ácido pipemídico (100\%) y cefuroxima (90.91\%), amoxiciina clavulanato (81.25\%), ampicilina (78.57\%) y cefalotina (72.22\%), aunque mantiene buena sensibilidad para imipenem (76.92\%), cefepime (72.73\%), amikacina (72.41\%), nitrofurantoína (70.37\%), así como a ceftriaxona (63.79\%) y ceftazidima (61.11\%) entre otros $(12,16)$.

Los mayores porcentajes de resistencia se observaron para amoxicilina (78,87\%) y cefalexina (71,83\%). La nitrofurantoina, fosfomicina, fluoroquinolonas y algunos betalactámicos, todavía representan una alternativa de utilidad en la quimioterapia de las infecciones urinarias no complicadas en pacientes diabéticos $(7,9,10)$

\section{CONCLUSIÓN.}

Los pacientes diabéticos son más propensos a padecer infección de vías urinarias que un paciente no diabético. Es más común en mujeres que en hombres, esto puede deberse a infecciones vaginales. Se observó que los pacientes con más de 5 años de haberse diagnosticado como diabéticos fueron ligeramente más propensos a sufrir de infección de vías urinarias.

Los pacientes diabéticos son más propensos a padecer infección de vías urinarias que un paciente no diabético. Más frecuente en mujeres; los malos hábitos de higiene, la presencia de cálculos renales y una vida sexual activa resultaron factores relacionados para las infecciones urinarias. Un tiempo de enfermedad mayor de 10 años, la insuficiencia renal crónica, litiasis renal, vejiga neurogénica, uso de corticoides, infección urinaria previa y presentar infección vaginal o patología prostática están asociadas con la ocurrencia de infección urinaria en la población de diabéticos.

El síntoma más frecuente es la fiebre. Los síntomas del tracto urinario presentes en el de pacientes diabéticos, son: La incontinencia de esfuerzo, de urgencia seguida por síntomas obstructivos e irritativos.

Se aislaron con más frecuencia gérmenes gram negativos, de los cuales predominó Escherichia coli, seguido de Enterobacter y Klebsiella pneumoniae. Gérmenes fueron gram positivos, estafilococos.

La resistencia de E. coli fue elevada contra clindamicina, ácido nalidíxico, ácido pipemídico, cefuroxima, amoxiciina/clavulanato, ampicilina y cefalotina; buena sensibilidad para imipenem, cefepime, amikacina, nitrofurantoína, ceftriaxona y ceftazidima, entre otros 


\section{REFERENCIAS BIBLIOGRÁFICAS}

1. García LA, Deveze MA, Alba C, Mendoza CL, Ruiz AJ, Zapata JR. Evaluación de la función renal en pacientes diabéticos: Relación albúmina/creatinina, riesgo litogénico e infección urinaria. Verano de la Investigación Científica. México 2017; 3(2): 376-380. http://www.jovenesenlaciencia.ugto.mx/index.php/jo venesenlaciencia/article/view/1739

2. Molina EM, Salas GP. Perfil de sensibilidad y resistencia antibiótica en mujeres diabéticas tipo 2 con infección del tracto urinario por Escherichia coli. Hospital Vicente Corral Moscoso, Cuenca 2014-2016 [Tesis Pre-Grado]. Ecuador: Universidad de Cuenca. Facultad de Ciencias Médicas; 2017. http://dspace.ucuenca.edu.ec/bitstream/123456789/ 28253/1/PROYECTO\%20DE\%20INVESTIGACI\%c3\%93 N.pdf

3. Mota JA. Gérmenes en infecciones del tracto urinario en pacientes diabéticos. Ciudad Hospitalaria "Dr. Enrique Tejera" enero-mayo 2015 [Tesis Pre-Grado]. Venezuela: Universidad de Carabobo. Facultad de Ciencias de la Salud; 2015.

http://mriuc.bc.uc.edu.ve/bitstream/handle/12345678 9/3213/Jmota.pdf?sequence $=1$

4. Sharma S, Govind B, kumar S, Kinjarapu S, Rasool M. Clinical and laboratory profile of urinary tract infections in type 2 diabetics aged over 60 years Journal of clinical and diagnostic research. India 2017; 11(4): 25-28.

https://www.ncbi.nlm.nih.gov/pubmed/28571186

5. Jagadeeswaran G, Zeya M, Rajangam T. Urinary Tract Infection in Diabetics: A five-year retrospective study on the prevalence of bacterial isolates and its antibiotic susceptibility patterns in a tertiary care hospital in South India. International Journal of Contemporary Medical Research. India 2018; 5(4): 33-38.

https://www.ijcmr.com/uploads/7/7/4/6/77464738/ijc

6. mr_1999_v2.pdf

Gordillo F, Barrera F. Perfil de resistencia de uropatógenos en pacientes con diabetes en Quito, Ecuador, inquietante panorama. Salud pública de México. Ecuador 2018; 60(1): 97-98.

http://www.scielo.org.mx/pdf/spm/v60n1/0036-3634

7. -spm-60-01-97.pdf

Lucas E, Cristóbal FQ, Castellano M. Infección urinaria en pacientes con diabetes mellitus tipo 2: Frecuencia, etiología, susceptibilidad antimicrobiana. Kasmera. 2019; 46(2): 139-152.

https://go.gale.com/ps/anonymous?id=GALE\%7CA58 $2622133 \&$ sid $=$ googleScholar $\& v=2.1 \& i t=r \& l i n k a c c e s$

8. $s=a b s \& i s s n=00755222 \& p=I F M E \& s w=w$

Nocua LC, Cortés JA, Leal AL, Fitzgerald G, Ovalle MV, Saavedra SY, et al. Perfil de sensibilidad antimicrobiana de microorganismos causantes de infecciones urinarias en pacientes con diabetes mellitus en Colombia. Biomédica. Colombia 2017; 37(3): 353-60. http://www.scielo.org.co/pdf/bio/v37n3/0120-4157bio-37-03-00453.pdf

9. Erazo PN, Mejía CP. Características de resistencia bacteriana a los antibióticos en pacientes con Diabetes Mellitus tipo 2 con infección del tracto urinario en el servicio de clínica del Hospital "Luis F. Martínez" durante el período 2012-2017 (Tesis Pre-Grado). Ecuador: Universidad de Cuenca. Facultad de Ciencias Médicas; 2018.

http://dspace.ucuenca.edu.ec/handle/123456789/3 1629

10. Aswami SM, Chandrashekar KN, Pruthvi BC. Clinical profile of urinary tract infections in diabetics and non-diabetics. Australas Med J. 2014; 7(1): 29-34 https://www.ncbi.nlm.nih.gov/pmc/articles/PMC392 0469/

11. Garg V, Bose A, Jindal J, Goyal A. Comparison of Clinical Presentation in Diabetic and Non-Diabetic Females with Urinary Tract Infection Assessed as Per the European Association of Urology Classification. J Clin Diagn Res. 2015; 9(6): 12-14.

https://www.ncbi.nlm.nih.gov/pmc/articles/PMC452 5549/

12. Tovar H, Barragan B, Sprockel J, Alba M. Infección del tracto urinario en pacientes hospitalizados con diabetes tipo 2. Revista Chilena de Endocrinología y Diabetes. Colombia 2016; 9(1): 6-10. http://www.revistasoched.cl/1_2016/2.pdf

13. Jiménez J, Carbajal A, Meza H, Moreno J, Serrano E. Prevalencia de síntomas urinarios en mujeres con diabetes mellitus. Revista Médica del Instituto Mexicano del Seguro Social. México 2016; 54(1): 70-74.

https://www.medigraphic.com/pdfs/imss/im-2016/i m161l.pdf

14. Batista A, De la Paz W, Baldera J, Castro R, García Y. Frecuencia de infección de vías urinarias en pacientes diabéticos asistidos en un centro rural. Revista Médica Dominicana. República Dominicana 2013; 74(2): 33-34.

https://studylib.es/doc/7675861/revista-m\%C3\%A9 dica-dominicana---colegio-m\%C3\%A9dico-dominic ano

15. Ríos MV. Características sociodemográficas, clínicas y antecedentes patológicos asociados a infección del tracto urinario en diabéticos: estudio de casos y controles (Tesis Post-Grado). Perú: Universidad Nacional Federico Villareal. Facultad de Medicina; 2018.

http://repositorio.unfv.edu.pe/handle/UNFV/2186

16. Coila J. Etiología y susceptibilidad antimicrobiana de uropatógenos en pacientes diabéticos con infección urinaria en el Hospital May. Od. Julio Pinto Manrique, Arequipa - 2014 [Tesis Pre-Grado]. Perú: Universidad Católica de Santa María. Facultad de Medicina Humana; 2015.

http://tesis.ucsm.edu.pe/repositorio/bitstream/hand 


\section{REFERENCIAS BIBLIOGRÁFICAS}

le/UCSM/3222/70.1992.M.pdf?sequence=18isAllowe $d=y$

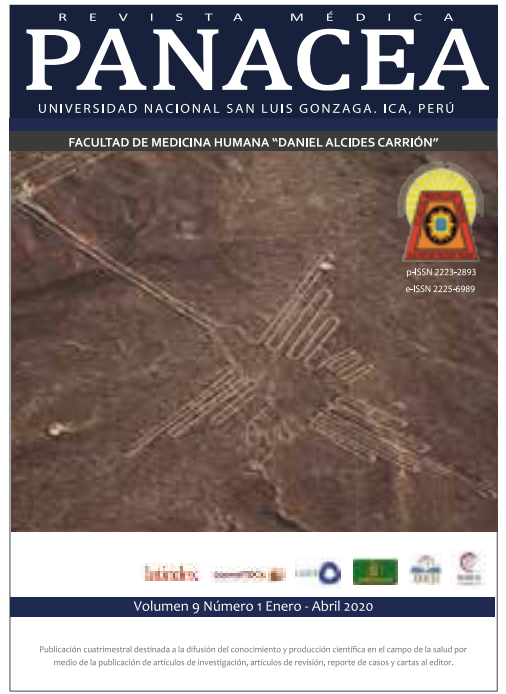

